\title{
A Dynamic Agents' Behavior Model for Computational Trust
}

\author{
Maria Joana Urbano, Ana Paula Rocha, and Eugénio Oliveira \\ LIACC / Departamento de Engenharia Informática \\ Faculdade de Engenharia, Universidade do Porto \\ Rua Dr. Roberto Frias, 4200-465 Porto, Portugal \\ \{joana.urbano, arocha, eco\}@fe.up.pt
}

\begin{abstract}
The development of computational trust models is growing in attention in the community of multi-agent systems and these models are currently seen as of extreme importance in social networks, electronic business and grid computing, among others. However, one of the biggest limitations in validating the existing computational trust models is the absence of realistic models of the behavior of agents. In fact, most of the work done in this area assumes that agents behave following simple and static probabilistic models. In this paper, we present a formal model of behavior of business agents that entail in inter-organizational exchanges, taking as basis diverse literature on socio-economic theories. With this model, we empirically show that some of the computational trust approaches which are more cited in the literature are not able to capture the temporal dynamics in the behavior of the business agents. Based on the results obtained from this study, we enumerate different properties that must be present in computational trust models in order to couple with realistic agents' behavior.
\end{abstract}

Keywords: Computational Trust, Behavior Models.

\section{Introduction}

Trust is a complex issue that is being studied in several areas of research, from sociology and psychology to economics and computer science. In computer science, computational models of trust and reputation are being proposed in order to support several processes associated with the decision making in social networks, electronic business and distributed resources. Namely, processes that imply the contact with strangers, where uncertainty and vulnerability, as well as the associated risk, are high.

In the concrete case of business exchanges, uncertainty is hard to reduce through personal relations. In the same way, vulnerability is also hard to reduce due to the presence of power relations [2] and to the existence of information asymmetry. Uncertainty and vulnerability leads to opportunism, which, in the words of Wathne and Heide (2000), can be defined as "some form of cheating or undersupply relative to an implicit or explicit contract" [14.

L. Antunes and H.S. Pinto (Eds.): EPIA 2011, LNAI 7026, pp. 536 550 2011.

(C) Springer-Verlag Berlin Heidelberg 2011 
The opportunism problem in interfirm relationships is widely studied in economics literature and different governance mechanisms are proposed to manage opportunism, from specific forms of control and monitoring to selection mechanisms, that may include certification and reputation.

Therefore, the behavior of buyers and suppliers engaged in interfirm exchanges is built from a difficult balance between several complex factors that include trust, risk, opportunism and power. In order to estimate the trustworthiness of a potential partner to an exchange, computational trust models that infer future behaviors from past evidence must take these factors into account. However, many of these models tend to aggregate the past evidence using simple statistical-based techniques. Besides, as they are usually empirically evaluated using simple statistical models of agents' behavior, it is difficult to extrapolate their performance to real situations.

In this paper, we give further insight on this issue, by proposing a model of behavior for business agents participating in interfirm purchasing activities. Although simple, this model is based on important concepts associated with trust in interfirm relationships, and incorporates behavioral dynamics that are not present in statistical-based models used in most of the computational trust research.

Then, we evaluate three representative computational trust approaches using our model of behavior, and show that these approaches fail to capture the dynamics of trust building and maintenance. As a results of this study, we pinpoint characteristics that we think must be present in computational trust models in order for them to be applied in real scenarios.

This paper is structured as follows: Section 2 presents related work on computational trust models and on the models of behavior used to validate these computational approaches. Section 3 presents the model of behavior that we have developed taking into consideration diverse literature on trust in interfirm relationships. In Section 4, we perform an empirical analysis of the limitations of current computational trust models when addressing models of dynamic behavior of agents that engage in interfirm relationships. Finally, Section 5 concludes the paper.

\section{Related Work}

There are several computational models of trust. Some of the more cited in the literature are able to estimate the trustworthiness of the agents in evaluation by aggregating past evidence on these agents using simple statistical techniques. Paradigmatic examples of these models are REGRET [9] and FIRE [3], which compute the direct trust on an agent weighing all the existent evidence on the agent according to their recency.

The evaluation of these models is done using relative simple models of the behavior of agents. In 3, the testbed built to evaluate the FIRE approach is a multi-agent system consisting of agents that provide services and agents that consume these services. It considers three different types of providers, whose 
performance level vary within a range of five possible values with a given standard deviation. Furthermore, there is a direct mapping between these values of performance and the utility gained by the consumers. Although the model allows for some dynamics (e.g. at every round, the suppliers can change the average level of performance by a given amount or even switch to a new profile), it is however a pure statistical model and does not reflect known theories of behavior in consumer-provider scenarios.

TRAVOS [7] and The Beta Reputation System [5] are other well known models that use Beta distributions to aggregate trust evidences. In TRAVOS, the behavior of an agent acting as a provider is given by a probability that it will participate in a successful interaction (trustworthy behavior) and a probability that it will perform an unsuccessful interaction (untrustworthy behavior). This behavior governs the tendency of an agent to fulfill or default on its obligations to the other party in the interaction.

More recently, a new trend of situation-aware computational trust models has appeared. These models also rely on past evidence of the agents in evaluation to compute their trustworthiness, however here every piece of evidence is assigned a different level of importance taking into account the similarity of its context with the current situation under evaluation. One of these models is the Context Space and Reference Contexts model (CSRC), presented in 86. This approach defines, for each one of the agents in evaluation, a multi-dimensional context space representing the possible situations in evaluation. When aggregating the past evidence, it calculates the similarity of the context of each evidence with the context of the situation in evaluation, and uses the level of similarity to weight the relevance of the piece of evidence being aggregated. This model is evaluated in an interesting scenario where humanitarian aid organizations require transportation services from local transporters after a major disaster. The selection of the transporters is based on their bid prices and on their trustworthiness. The transporters are modeled with bid prices based on transportations costs and profit margins and on their competence in specific scenarios. However, this represents a static behavior that does not allow the agents in evaluation to evolve with time.

Finally, the model presented in [13] is also situational, however it works in a rather different way than the CSRC model. It is constituted by a heuristicbased aggregator (Sinalpha) and a situation-aware tuner (Contextual Fitness). The model is evaluated in an electronic commerce scenario where supplier agents have fixed handicaps in performing specific context-based tasks. Once again, this model of behavior is probabilistic and does not evolve over time.

\section{The Model}

In this section, we present a model of behavior for agents that assume the roles of buyers and suppliers in buyer-supplier relationships. This model is based essentially on literature on trust in interfirm relationships. 
Due to the complexity of the thematic, and also to the absence of consensual definitions and approaches among the different branches of research that study this issue, we opted to perform a significant amount of simplifications and hard assumptions. Nevertheless, the model is sufficiently expressive to allow for realist dynamics in the agents' behavior and to permit behaviors that evolve over time. Therefore, its utilization is adequate to the purpose of this paper, which consist in testing our suspicion that current computational trust are inadequate in capturing the dynamics of behavior of the agents in evaluation.

\subsection{Background}

The scenario underlying our model is an Electronic Institution (EI) where buyersupplier relationships are developed between firm agents registered in the institution. In this scenario, buyer agents announce their business needs at a regular pace and supplier agents answer by sending a proposal. The buyers then select the best proposals by weighing up the utility associated to each proposal with the trustworthiness of the proponent agent. Finally, a simple purchasing contract - stipulating the good to deliver and the associated quantity, price, and delivery time conditions - is automatically drafted by the EI to regiment the exchange interaction.

The EI has limited monitoring capabilities. More specifically, for each contract established, the institution controls if the delivery time is respected by the supplier. When the transaction is over, the EI builds a contractual evidence, which includes the outcome $o$ of the transaction $(o=f$ when the good is provided on time, $o=f d$ when the delivery is delayed, and $o=v$ if the good is not delivered at all). Other costly monitoring activities (e.g. about the quality of delivered products) are not performed by the EI or by any one of the corporate agents registered in the EI.

\section{$3.2 \quad$ Agents}

There are two types of agents: the ones that play the role of buyers, and the ones that play the role of suppliers (Equation 1). In this section, we start by describing the latter, and then proceed to the description of buyers.

$$
\text { Agents }=\text { Buyers } \cup \text { Suppliers }
$$

Supplier Agents. These agents are characterized by the following properties: dimension, reciprocity, subcontracting power and willingness to promote goodwill.

In the scope of this paper, the dimension property measures the recent contractual activity of the supplier, i.e. the ratio of the number of contracts won by the supplier in the recent past to the number of proposals it had made in the same period of time. The contractual activity of a supplier may vary through the agent's life, due to several factors (e.g. its current trustworthiness, the utility 
of the proposals it makes, and whether or not the agent is engaged in goodwill relationships). Hence, the dimension of supplier agents may evolve over time, switching from small to big and vice-versa. Equation 2 shows the properties that identify the dimension of a supplier. In the Equation, CAThreshold is a threshold parameter that can be configured in the experiments.

$$
\begin{aligned}
& \text { ContractualActivity }(x)<C \text { AThreshold } \Rightarrow \operatorname{Small}(x) \\
& \text { ContractualActivity }(x) \geqslant C \text { AThreshold } \Rightarrow \operatorname{Big}(x)
\end{aligned}
$$

The reciprocity property indicates the ability of the supplier agent to reciprocate to the buyer in current contract when the latter shows goodwill towards the former. The subcontracting power property indicates the ability/willingness of the supplier to subcontract when the current order made by the buyer is bigger than the supplier's current capacity to fulfill it, i.e., when the supplier is small. Finally, the willingness to promote goodwill property is related with the previous property. It indicates the willingness of the supplier agent to subcontract when the current order made by the buyer is bigger than the supplier's current capacity to fulfill it, even when the buyer has not shown goodwill towards it.

According to the aforementioned properties, we define three distinct categories of suppliers by providing the necessary and sufficient conditions for membership through predicates' formulas, as shown in Equation 3.

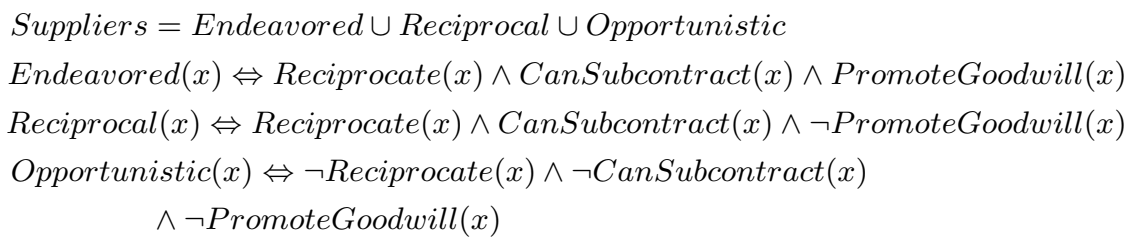

We are now in conditions to model the behavior of supplier agents. This behavior is defined in terms of the ability and willingness of the supplier to provide the good to the buyer within the deadline stipulated in the contract.

The decision of whether to delay or not the delivery as stated in current contract takes into consideration the category of the supplier (as defined in Equation 3), its current dimension (cf. Equation 2), the size of the order, and the agent's belief about the goodwill motivation of the buyer towards it.

Equation 4 illustrates the behavior of suppliers of type Endeavor concerning the property of delaying the delivery of goods. These agents normally do not delay contracts. They tend to subcontract every time they get a contract that is bigger than their current capacity, even if they loose some utility in the short term due to subcontracting 11 In other words, they show an attitude of goodwill towards the buyer seeking a longterm relationship with it. However, as these suppliers grow in dimension and are eventually considered big, they delay the delivery of small contracts if the corresponding buyers have not showed any goodwill towards them.

\footnotetext{
${ }^{1}$ In this model, we do not model the loss of utility of suppliers due to subcontracting.
} 


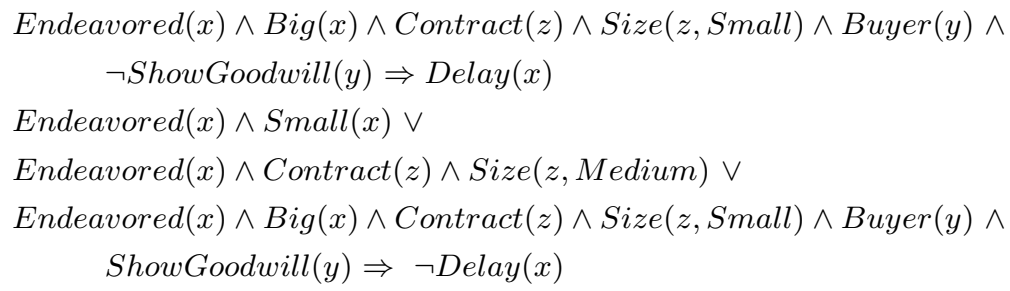

Equation 5 shows the behavior of suppliers of type Reciprocal. There are two situations where the suppliers may delay the delivery to the buyer: when the size of the order is bigger than their current supplying capacity, and when they are considered big suppliers and the current order is of small magnitude. In both situations, these suppliers opt to not delay the delivery (even if it implies that some subcontracting must be done, in the first case) if the corresponding buyers have already shown goodwill attitudes towards them. Otherwise, they do delay these deliveries.

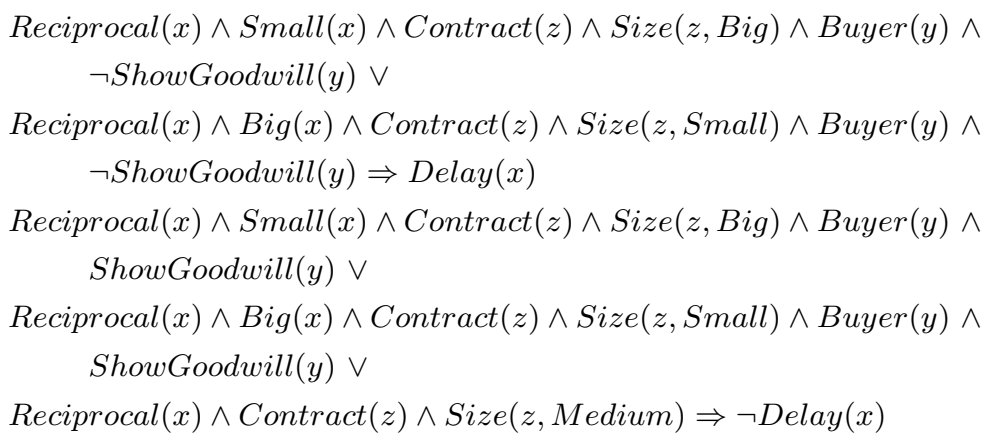

Finally, Equation [6 illustrates the behavior of the last category of suppliers, Opportunistic. This behavior reflects the fact that these suppliers do not have subcontracting power ability.

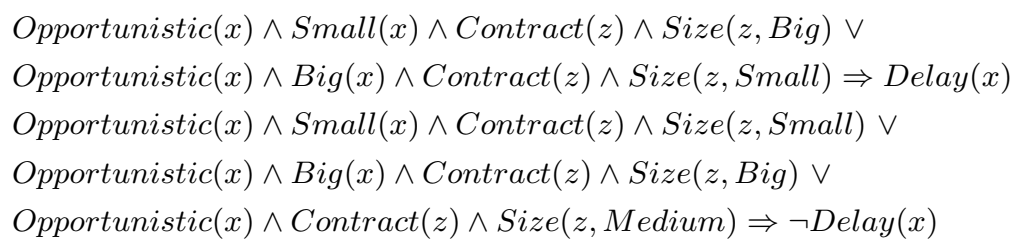

Buyer Agents. In the previous section, we showed that supplier agents base their delivery decisions taking into account their own idiosyncrasies and contextual information that they sense in the environment. In this section, we describe the behavior of buyer agents taking into account how they react to a delay in delivery. Buyer agents can be classified as Benevolent or NonBenevolent, taking 
into consideration two different properties: reciprocity and ability to promote goodwill relationships (cf. Equation 7).

$$
\begin{aligned}
& \text { Buyers }=\text { Benevolent } \cup \text { NonBenevolent } \\
& \text { Benevolent }(x) \Leftrightarrow \text { Reciprocate }(x) \wedge \text { PromoteGoodwill }(x) \\
& \text { NonBenevolent }(x) \Leftrightarrow \neg \text { Reciprocate }(x) \wedge \neg \text { PromoteGoodwill }(x)
\end{aligned}
$$

The reciprocity property indicates whether or not the buyer will reciprocate to existent manifestations of goodwill from suppliers. The ability to promote goodwill property indicates the propensity of the buyer to initiate relationships based on reciprocity that eventually will lead to goodwill.

Given the categories of the buyers, we are now in conditions to describe their behavior. This latter translates in decisions about whether the buyers will denounce, or not, a contract whose delivery is delayed (Equation 8) 20 As can be observed in Equation 8 , in the case of buyers of type Benevolent, the decision to denounce the delay depends on whether or not the buyer has already established a goodwill relationship with the supplier.

$$
\begin{aligned}
& \text { Benevolent }(x) \wedge \text { Supplier }(y) \wedge \operatorname{Delayed}(y) \wedge \operatorname{HasGoodwill}(x) \Rightarrow \neg \operatorname{Denounce}(x) \\
& \text { Benevolent }(x) \wedge \operatorname{Supplier}(y) \wedge \operatorname{Delayed}(y) \wedge \neg \operatorname{HasGoodwill}(x) \vee \\
& \text { NonBenevolent }(x) \wedge \operatorname{Supplier}(y) \wedge \operatorname{Delayed}(y) \Rightarrow \operatorname{Denounce}(x)
\end{aligned}
$$

Although the behavior of buyers is defined in simpler terms than the behavior of suppliers, it also allows for important dynamics in the behavior of agents.

\subsection{Theoretical Motivation of the Model}

In the previous section we gave an intuitive description of the agents' behavior model. We are now ready to present the main theoretical socio-economic notions underlying this model.

Electronic Institutions, Contract Drafting and Monitoring. In our work we use the paradigm of electronic institutions (EI). These institutions, which can be seen as an umbrella for different types of virtual organizations, strategic alliances and organizational networks, are indeed a promising trend for interorganizational relationships 1 .

The contracts established in the EI lack the formal detail. This is common within the textile industry, where contracts can even be relational [11]. Moreover, designing detailed contracts involves substantial drafting costs [14] if it is not considered impossible [15]. Also, the EI has limited monitoring functionalities. Mechanisms for control and coordination are costly and we use instead trust as a complementary control mechanism, as suggested in [4.

\footnotetext{
${ }^{2}$ When a supplier does not deliver within the deadline, the EI registers a delay. However, it is up to the buyer to denounce this delay (resulting in outcome $o=v$ ) or to be benevolent with it $(o=f d)$.
} 
Finally, we assume that buyer agents are able to access information about all contracts established in the electronic institution. There is not enough empirical knowledge supporting this assumption. However, we verify similar concepts in highly, though open, regulated markets, such as the stock market exchange, where the information about firms is made public. Moreover, an agent that is evaluating a business party may acquire information about contracts established between the party and other agents through reputation, opinions and recommendations. The way that this information is acquired and its reliability does not affect the aggregation models, per se, and is out of the scope of this paper.

Behavior of Agents. The behavior of suppliers is based on the study of opportunism. Following Wathne and Heide (2000), opportunism can be passive or active and applies under existent or new conditions (either present in an informal agreement or in a legal contract) 14. In existent conditions, it takes the form of evasion of obligations (passive) and violation (active), and under new conditions it takes the form of refusal to adapt to new circumstances (passive) and forced renegotiation (active).

In our work, we adopt the notion of passive opportunism under existent conditions, where obligations can be evaded. This can happen in two distinct situations. In the first situation, small suppliers bid to provide big quantities, even when they know that they are not able to fulfill what was stipulated in the contract. This is the case for suppliers of type Opportunistic and for suppliers of type Reciprocal in specific conditions. A common problem in interfirm relationships that is reflected in the proposed categorization is adverse selection, where suppliers hide their true attributes from the buyer. This happened in the famous Ford vs. Lear case, where Lear committed to supply the seats for all Ford Taurus versions, withholding the information about its lack of adequate resources. As a result, "Lear missed deadlines, failed to meet weight and price objectives, and furnished parts that did not work (Walton 1997)" and Ford incurred in substantial transaction costs [14.

Reciprocation and Goodwill Trust. As mentioned by D. Ireland and Webb (2007), when unanticipated contingencies surface, business partners may opt to show some goodwill towards the target agent, or to select tougher forms of action, depending on the magnitude of the contingency and the level of trust existing between the partners [4]. As described by Sako (2002), goodwill trust is a form of trust that develops within long-term relationships through repeated exchanges [10. It can be built upon reciprocation of initial favors that allow the establishment of the norms and shared values that characterize relational behavior and continued interactions between the partners (Jones (2001), referenced in [4]).

Dimension of Buyers and Suppliers. The dimension of buyers and suppliers is oversimplified in our model. Other properties may be added in the future, such as those derived from the complex concept of power in business relations. 


\section{Empirical Analysis}

This section describes the empirical study we have performed on the adequacy of existent computational trust models in capturing the dynamics of behavior that exist in interfirm relationships, as modeled in the previous section. In this study, we analyzed three distinct computational trust models, as described in the next section.

\subsection{Computational Trust Models}

The first model we analyzed was FIRE, more concretely, its component of aggregation of both direct experiences and reputation [3]. We chose this model because it is widely cited in the literature and also because it includes in its formula a temporal dimension given by weighing by recency. Therefore, it could be a good choice for dealing with populations whose behavior evolves over time. The formula used in the experiments to estimate the trustworthiness of agents $a g$ is depicted in Equation 9. In this formula, $w_{i}$ is the weight of current evidence, $v_{i}$ is the value of the outcome of the evidence and $\Delta_{i}$ is the time elapsed between the occurrence of the evidence and current time.

$$
\operatorname{trustworthiness}(\mathrm{ag})=\frac{\sum_{i=0}^{N-1} w_{i} \cdot v_{i}}{\sum_{i=0}^{N-1} v_{i}}, w_{i}=e^{\frac{-\Delta t_{i}}{\lambda}}, \lambda=\frac{-0.5}{\ln (0.5)}
$$

The second model we analyzed was the situation-aware CSRC model [8] referred to in the related work. As FIRE, it also aggregates evidences using weighting means. However, this model weights the evidences by the similarity between the context of the evidence being aggregated and the context of the current situation in assessment (cf. Equations 10, 11] and 12).

$$
\begin{gathered}
\text { trustworthiness }(a g, s)=\frac{\sum_{i=0}^{N-1} w_{i} . v_{i}}{\sum_{i=0}^{N-1} v_{i}}, w_{i}=e^{-d\left(c_{1}, c_{2}\right)} . \\
d^{\text {fabric }}\left(c_{1}, c_{2}\right)=\left\{\begin{array}{l}
0, \text { if fabric } c_{1}=\text { fabric } c_{2}, \\
1, \text { if fabric } c_{1} \neq \text { fabric } c_{2} .
\end{array}\right. \\
d^{\text {attr }}\left(c_{1}, c_{2}\right)=\mid \ln \left(\text { attr }_{1}\right)-\ln \left(\text { attr }_{2}\right) \mid .
\end{gathered}
$$

Finally, we chose as the third model another situation-aware approach (which we name here SACF), which includes the heuristic-based aggregator Sinalpha 12 . and the situation-aware tuner, Contextual Fitness [13. We chose to evaluate this model because it extracts tendencies of failure in an incremental way and therefore, as happened with the first two approaches, it could be a good choice to tackle the problem of dynamic populations of agents. Equations 13 and 14 show the main formulas of this model. The algorithm underlying $C F(a g, s)$, as well as a detailed explanation of Sinalpha's parameters, are documented in [13]. 


$$
\begin{gathered}
\text { trustworthiness }(a g, s)=\operatorname{sinalph} a(a g) * C F(a g, s), \\
\operatorname{sinalph} a(\alpha, a g)=\delta \cdot \sin \alpha+\delta, \alpha=\alpha+\lambda \times \omega .
\end{gathered}
$$

\subsection{Testbed}

All experiments described in this paper were performed using the Repast simulation tool [6]. We ran three different experiments, one for each computational trust model considered.

At every round, a fixed number of buyer agents selected the best suppliers of textile fabric using a simple one round, multi-attribute negotiation protocol. Every buyer had a business need randomly assigned at setup, consisting of a fabric and associated values of quantity, price and delivery time.

The set of possible fabrics is given by $\{$ cotton, chiffon, voile $\}$. The values of quantity, price and delivery time are assigned randomly from sets $\{q \in \mathbb{N}: q \in$ $\left.\left[v_{\text {quant }, \text { min }}, v_{\text {quant }, \text { max }}\right]\right\},\left\{p \in \mathbb{N}: p \in\left[v_{\text {price, } \min }, v_{\text {price, max }}\right]\right\}$ and $\{d \in \mathbb{N}: d \in$ $\left.\left[v_{d t i m e, \min }, v_{d t i m e, \max }\right]\right\}$, respectively. The values $v_{i, \min }$ and $v_{i, \max }$ define the minimum and maximum values allowed in the simulation for each attribute $i$, respectively.

All suppliers registered in the EI were able to provide any type of fabric. When a buyer announced its need (in the form a call for proposals - cfp) to a defined set of suppliers, each one of these suppliers generated a proposal with its own values for the quantity, price and delivery time attributes. These values were generated randomly following a uniform distribution in the range $\left[v_{i, p, \min }, v_{i, p, \max }\right]$, where $v_{i, p, \min }$ and $v_{i, p, \max }$ are defined in Equation 15.

$$
\begin{aligned}
& v_{i, p, \min }=\max \left((1-\zeta) \times v_{i, c f p}, v_{i, \min }\right), \\
& v_{i, p, \max }=\min \left((1+\zeta) \times v_{i, c f p}, v_{i, \max }\right) .
\end{aligned}
$$

In Equation 15. $v_{i, c f p}$ is the value defined in the $c f p$ for attribute $i$ (quantity, price or delivery time), and $\zeta$ is a dispersion parameter that allows to define how distant the generated proposal is from the preferences of the buyer, as stated in the $c f p$.

Selection Strategy. After receiving the proposals from the suppliers, the buyer calculated the utility of each one of them. The utility of a proposal, $\mu_{p} \in[0,1]$, was given by the complement of the deviation between the client preferences specified in the $c f p$, for all the negotiable items price, quantity and delivery time, and what is offered in the received proposal (cf. Equation [16).

$$
\mu_{p}=1-\frac{1}{k} \times\left(\sum_{i}^{k} \frac{\left|v_{i, c f p}-v_{i, p}\right|}{v_{i, \max }-v_{i, \min }}\right) .
$$

In Equation 16, $v_{i, p}$ is the value of the negotiation attribute $i$ of the current proposal in evaluation and $k$ is the number of negotiation attributes considered in this paper. 
After calculating the utilities of all received proposals, the buyer selected the best proposal, by sorting the proposals by the weighted sum of their utility and the trustworthiness of the corresponding proponents, and by choosing the one that presented the highest value for this weighted sum. This assumes that, previous to the evaluation phase, the buyer estimated the trustworthiness of all suppliers that presented a proposal using one of the computational trust algorithms described above. Equation 17 illustrates the weighted sum, where $\tau$ stands for the estimated trustworthiness value and the weighting parameter $\omega_{\tau} \in[0,1]$ allows to configure the importance assigned to the trustworthiness component in the selection.

$$
\omega_{\tau} \times \tau+\left(1-\omega_{\tau}\right) \times \mu_{p}
$$

Finally, after the selection of the best proposal, the buyer establishes a contract with the selected supplier, stipulating that the latter must provide the fabric at the conditions of quantity, price and delivery time described in its proposal 3

Configuration Parameters. In all experiments, we used 10 buyer agents and 20 supplier agents. The behavior of these agents was extensively described in Section 3.2. Each experiment was composed of 30 episodes, and at every episode each buyer started a new negotiation cycle by issuing a new $c f p$. At the first episode of each experiment, the repository of trust evidences was cleaned, which means that the trustworthiness of all suppliers was set to zero. Finally, we ran every experiment 10 times.

Additional configuration parameters can be seen in Table 1 .

\subsection{Evaluation Metrics}

In these experiments, we considered five different performance metrics. The first metric was the utility of the transaction, $\mu_{t} \in[0,1]$, given by Equation 18, which was further averaged over all buyers and all episodes.

$$
\mu_{t}=\left\{\begin{aligned}
\mu_{p}, \text { if } o=f \\
0, \text { if } o=v
\end{aligned}\right.
$$

The second metric was the number of positive outcomes $\left(o^{+} \in[0,1]\right)$ obtained by a buyer agent in an episode, averaged over all buyers and all episodes. The third metric was the number of different suppliers $\left(\Delta_{\text {sup }} \in[0,1]\right)$ selected by all buyers in one episode, averaged over all episodes. Finally, the fourth and the fifth metrics measured the trustworthiness of the supplier and the utility of the proposal selected by a buyer in one episode $\left(\tau_{s} \in[0,1]\right.$ and $\mu_{s} \in[0,1]$, respectively), averaged over all buyers and all episodes.

\footnotetext{
${ }^{3}$ The negotiation mechanism we present in this paper is deliberately simple, as it does not constitute the focus of this work. We assume that the conclusions derived from our study using this mechanism are still valid in the presence of others, more complex negotiation protocols.
} 
Table 1. Configuration parameters

\begin{tabular}{c|c|c}
\hline parameter & value & appears in \\
\hline \hline$\zeta$ & 1.0 & Eq. [15] \\
$v i$ & $0.0(o=f), 0.5(o=f d), 1.0(o=v)$ & Eq. [9] \\
$\delta$ & 0.5 & Eq. [14] \\
$\lambda$ & $1.0(o=f), 0.5(o=f d),-1.5(o=v)$ & Eq. 9$]$ \\
$\omega$ & $\pi / 2$ & Eq. [9] \\
$\omega_{\tau}$ & 0.5 & Eq. [17] \\
\hline
\end{tabular}

\subsection{Results}

Table2 presents the results obtained in the experiments for the metrics described in Section 4.3 .

Table 2. Results obtained in the experiments

\begin{tabular}{|c|c|c|c|c|c|c|}
\hline$\#$ & Trust Model & $\mu_{t}$ & $o^{+}$ & $\Delta_{\text {sup }}$ & $\tau_{s}$ & $\mu_{s}$ \\
\hline \hline 1 & $\begin{array}{c}\text { SACF } \\
\text { (stdev) }\end{array}$ & 0.64 & 0.80 & 0.37 & 0.75 & 0.79 \\
& $(0.06)$ & $(0.07)$ & $(0.03)$ & $(0.06)$ & $(0.01)$ \\
\hline 2 & $\begin{array}{c}\text { FIRE } \\
\text { (stdev) }\end{array}$ & 0.55 & 0.67 & 0.37 & 0.76 & 0.83 \\
$(0.06)$ & $(0.05)$ & $(0.06)$ & $(0.06)$ & $(0.02)$ \\
\hline 3 & $\begin{array}{c}\text { CSRC } \\
\text { (stdev) }\end{array}$ & 0.59 & 0.70 & 0.41 & 0.76 & 0.84 \\
$(0.05)$ & $(0.05)$ & $(0.05)$ & $(0.04)$ & $(0.01)$ \\
\hline
\end{tabular}

From the results presented in Table 2 we verified that the FIRE approach got the worse results, both in terms of utility of transaction $\left(\mu_{t}=0.55\right)$ and of positive outcomes $\left(o^{+}=0.67\right)$, even with the obtained values for the number of distinct suppliers and trustworthiness of the selected supplier being approximately the same as those of the model that performed best (SACF).

Also, we verified that, between the situation-aware approaches, the SACF got better results than the CSRC approach, both in terms of utility of transaction $\left(\mu_{t}=0.64\right.$ vs. $\mu_{t}=0.59$ 9 $)$ and of positive outcomes $\left(o^{+}=0.80\right.$ vs. $\left.o^{+}=0.70\right)$. This happened even with the latter allowing for a broader selection of partners than the former (CSRC: $\Delta_{\text {sup }}=0.41 \mathrm{vs.} \mathrm{SACF:} \mathrm{Delta} a_{\text {sup }}=0.37$ ), which in turn increased the utility of the proposals received by the buyers (CSRC: $\mu_{s}=0.84$ vs. SACF: $\left.\mu_{s}=0.79\right)$.

\subsection{Interpretation of the Results}

From the results depicted in the previous section, we verified that recency is not enough to capture evolving behavior of agents, as the FIRE model got the worse

\footnotetext{
${ }^{4}$ We used the two-sample t-test to infer about the difference in $\mu_{t}$ between SACF and FIRE and between SACF and CSRC. The test results were statistically significant at the $\alpha=0.05$ level of significance.
} 
results. We also verified that the dynamic extraction of failure tendencies of the SACF approach seemed to be more adequate to evolving behaviors than the use of reference contexts of the CSRC approach.

However, looking at the traces of the experiments, we observed that even the best approach (SACF) had a relatively poor performance in detecting the changes on the behavior of buyer and suppliers agents, based on reciprocation and goodwill relations that formed, and also by the evolving dimension of suppliers.

In order to clarify this question, we present next a simple trace extracted from the experiments with the SACF model, representing an evaluation performed by agent $C 0$ (of type Benevolent) to a supplier agent of type Recriprocate. At the evaluation time, agent $C 0$ had access to the following contractual evidences of the supplier in evaluation:

\begin{tabular}{cccc}
\hline episode client & cfp & outcome \\
\hline 1 & C2 & chiffon low medium & $\mathrm{f}$ \\
2 & $\mathrm{C} 2$ & chiffon low low & $\mathrm{f}$ \\
2 & $\mathrm{C} 3$ & voile medium big & $\mathrm{f}$ \\
3 & $\mathrm{C} 1$ & chiffon low medium & $\mathrm{f}$ \\
3 & $\mathrm{C} 2$ & chiffon medium low & $\mathrm{f}$ \\
3 & $\mathrm{C} 3$ & voile high medium & $\mathrm{v}$ \\
3 & C9 & chiffon low low & $\mathrm{f}$ \\
4 & C0 & voile low big & $\mathrm{f}$ \\
4 & $\mathrm{C} 2$ & chiffon medium big & $\mathrm{f}$ \\
4 & $\mathrm{C} 6$ & cotton low low & $\mathrm{v}$ \\
4 & $\mathrm{C} 7$ & voile low low & $\mathrm{v}$ \\
4 & $\mathrm{C} 9$ & chiffon medium medium & $\mathrm{f}$ \\
\hline
\end{tabular}

Using the SACF algorithm, the model was able to detect the following tendencies of failure: (cotton, $*, *),($ voile, $*$, low) and (voile, $*$, medium). Also, the algorithm inferred that the supplier had the following tendency to not delaying contracts: (chiffon, $*, *$ ) and (voile, $*$, big)

In this extreme case, when the number of evidences available is reduced, the model was not able to understand the shift that occurred in the dimension of the suppliers. In other situations (which traces we do not reproduce here), this model was not also able to detect goodwill relationships between the interfirm partners.

\section{Conclusions}

In this paper, we raised the suspicion that current computational trust models were not able to detect the dynamics of agents that engage in trust relationships. In order to confirm this suspicion, we presented a conceptual model for the behavior of trading agents that was based on existent literature on interfirm relationships. Although the presented model is simple, it is able to model 
evolving behaviors that are due to the establishment of reciprocation and goodwill relationships between business partners and to changes in the dimension of supplier agents.

Next, we evaluated three computational trust models, including the representative FIRE model that embeds the recency of the trust evidences on its algorithm and two situation-aware approaches. The results have shown that these latter models performed better than FIRE in evolving populations like the ones modeled in this paper. Also, we have observed that even the model that performed best was not able to detect the complex web of relations that happens between interfirm business partners.

From this study, we concluded that computational trust models must interpret the past evidence in light of the context where these evidences occurred. Also, the models must take into account the (inferred) relation between the partners to the exchange, as the outcome of the evidences may vary substantially depending on the evaluator and its relationship with the evaluated. Finally, computational trust models must process the past evidence through a temporal lens, and ideally they must infer the dynamics of behavior that evolve over time.

Acknowledgments. This research is supported by Fundação para a Ciência e a Tecnologia (FCT), under project PTDC/EIA-EIA/104420/2008. The first author is supported by FCT under grant SFRH/BD/39070/2007.

\section{References}

1. Bachmann, R.: Trust, power and control in trans-organizational relations. Organization Studies 22(2), 337-365 (2001)

2. Heimer, C.: Solving the problem of trust. In: Cook, K.S. (ed.) Trust in Society. Russell Sage Foundation Series on Trust, pp. 40-88 (2001)

3. Huynh, T.D., Jennings, N.R., Shadbolt, N.R.: An integrated trust and reputation model for open multi-agent systems. Autonomous Agents and Multi-Agent Systems 13, 119-154 (2006)

4. Ireland, R.D., Webb, J.W.: A multi-theoretic perspective on trust and power in strategic supply chains. Journal of Operations Management 25(2), 482-497 (2007); special Issue Evolution of the Field of Operations Management SI/ Special Issue Organisation Theory and Supply Chain Management

5. Ismail, R., Josang, A.: The beta reputation system. In: Proceedings of the 15th Bled Conference on Electronic Commerce (2002); paper 41

6. North, M.J., Howe, T.R., Collie, N.T., Vos, J.R.: A declarative model assembly infrastructure for verification and validation. In: Takahashi, S., Sallach, D.L., Rouchier, J. (eds.) Advancing Social Simulation: The First World Congress, Springer, Heidelberg (2007)

7. Patel, J.: A Trust and Reputation Model for Agent-Based Virtual Organisations. Ph.D. thesis, University of Southampton (2006)

8. Rehák, M., Pěchouček, M.: Trust modeling with context representation and generalized identities. In: Klusch, M., Hindriks, K.V., Papazoglou, M.P., Sterling, L. (eds.) CIA 2007. LNCS (LNAI), vol. 4676, pp. 298-312. Springer, Heidelberg (2007) 
9. Sabater, J., Sierra, C.: Regret: reputation in gregarious societies. In: Proceedings of the Fifth International Conference on Autonomous Agents, AGENTS 2001, pp. 194-195. ACM, New York (2001)

10. Sako, M.: Does trust improve business performance? (2002), http://hdl.handle.net/1721.1/1462

11. Tokatli, N.: Global sourcing: insights from the global clothing industry - the case of zara, a fast fashion retailer. Journal of Economic Geography (2007)

12. Urbano, J., Rocha, A.P., Oliveira, E.: Computing confidence values: Does trust dynamics matter? In: Lopes, L.S., Lau, N., Mariano, P., Rocha, L.M. (eds.) EPIA 2009. LNCS, vol. 5816, pp. 520-531. Springer, Heidelberg (2009)

13. Urbano, J., Rocha, A.P., Oliveira, E.: Trustworthiness tendency incremental extraction using information gain. In: Proceedings of the 2010 IEEE/WIC/ACM International Conference on Web Intelligence and Intelligent Agent Technology, WI-IAT 2010, vol. 02, pp. 411-414. IEEE Computer Society, Washington, DC (2010)

14. Wathne, K.H., Heide, J.B.: Opportunism in interfirm relationships: Forms, outcomes, and solutions. The Journal of Marketing 64(4), 36-51 (2000)

15. Williamson, O.E.: Transaction-cost economics: The governance of contractual relations. Journal of Law and Economics 22, 233-261 (1979) 